\title{
We Michel Guibal (1935-2017)
}

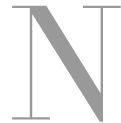

é à Paris le 5 décembre 1935, Michel Guibal, psychiatre et psychanalyste, excellent clinicien de la folie, grand lecteur d'Artaud et des surréalistes, est mort à Paris le 10 mars 2017. Issu d'un milieu populaire, il poursuit d'abord des études médicales, songeant à devenir chirurgien puis il s'oriente vers la psychiatrie. En 1964, élève de Henri Ey à l'hôpital de Bonneval, il découvre l'univers asilaire et décide d'aller plus loin dans sa carrière. Trois ans plus tard, il passe le concours des hôpitaux psychiatriques de la Seine et se retrouve à l'hôpital Sainte Anne. La même année, il assiste à une tragédie qui le marquera toute sa vie : le meurtre d'Yves Bertherat, à l'âge de 35 ans, par un malade mental. Poète, membre du comité de la revue Esprit, Bertherat était l'un des psychiatres les plus brillants de sa génération et l'événement sera répercuté dans toute la presse : “ Le dimanche 15 octobre 1967 à 7 heures du matin, à l'hôpital de Perray-Vaucluse, un malade a blessé à coups de révolver un infirmier; quelques instants plus tard il a tiré sur le Dr Y. Bertherat, Médecin-chef intérimaire du service, l'atteignant dans la région cardiaque de deux balles, puis il a blessé un de ses camarades; il a ensuite retourné l'arme contre lui-même. "Guibal entre à l'École freudienne de Paris, fondée par Jacques Lacan après une analyse sur le divan de Piera Aulagnier et un contrôle chez Gisela Pankow dont il suivra le séminaire pendant de très longues années et dont il sera l'un des héritiers majeurs en France. Passionné d'histoire de la psychanalyse, il adhère à la SIHPP dès sa création et il participe en 1981 avec son ami Jacques Nobécourt à la traduction française du dossier découvert par Aldo Carotenuto et Carlo Trombetta sur les relations entre Sabina Spielrein, Carl Gustav Jung et Sigmund Freud (Aubier). L'ouvrage obtient un beau succès. C'est alors qu'il rencontre le philosophe chinois Huo Datong venu à Paris pour étudier l'œuvre de Lacan. Il le prend en analyse et poursuit l'expérience en se rendant à son tour à plusieurs reprises à Chengdu où celui-ci fonde un groupe psychanalytique, le premier du genre en Chine, et commence à former des élèves. En 2000, Guibal sera l'un des participants les plus actifs des États Généraux de la psychanalyse. Hostile à tous les dogmatismes, il était connu pour son engagement permanent dans les combats qui ont marqué l'histoire de la psychanalyse et de la psychiatrie pendant un demi-siècle.

Élisabeth Roudinesco 


\section{We À Claude Guibal, son épouse et leurs enfants et leurs petits-enfants}

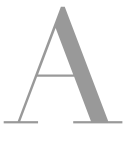

ux amis de Psychanalyse Actuelle.

Michel Guibal est décédé à Paris ce vendredi matin 10 mars 2017 à la Maison Jeanne Garnier où il était en soins palliatifs.

Il était...

Michel, Michel... Michel... des points de suspension, tu savais t'en servir. Ils sont à toi maintenant. Avec d'autres, on s'est suivis, toujours proches, jamais complices face à l'adversité du monde et de celle présente dans la psychanalyse surtout. Nous savons grâce à toi de quoi il s'agit désormais. Remonter le sens, le chemin du symptôme... aller contre, contre la masse compacte comme disait Freud... De Michel, sa manière, son originalité, son idée maitresse c'était quand il le fallait, de désobéir sans détruire. Jamais. Désobéir aux injonctions des maitres, celles et ceux qui se veulent tels entre psychanalystes. Et entre psychiatres, car venu de la psychiatrie asilaire il avait un lien privilégié à Henri Ey, pour beaucoup notre enseignant, c'était son appui dont tout récemment il nous avait entretenu abondamment avec son talent de conteur. Ce fut la dernière fois en octobre 2016 qu'il fit son séminaire à la demande de certains d'entre nous. Il est venu à Psychanalyse Actuelle dès le début en 1988. Il voulait participer à cette aventure pour faire entendre comment l'Europe nazifiée nous avait abîmés. Et atteint les liens des gens de la parole, de celles et ceux pour qui parler engage. D'où sa critique des institutions analytiques, d'où son intense intérêt pour la passe, soit le passage du privé au public du témoignage d'un praticien qui a en lui l'exigence de dire ce passage à l'analyste. Sans relâche, il questionne l'hétérogénéité de nos constructions d'associations pour praticiens de la psychanalyse. Ne jamais rester dans l'homogénéité trompeuse et ne pas prendre de risque et dès lors rester ignorants face aux novations de nouveaux venus. Et ce fut l'inter! L'inter-associatif européen de psychanalyse. L'I-AEP...

Avec de formidables échanges sur le trait d'union entre inter redoublé de ce petit trait d'écrit pour faire vivre et lutter pour que la différence se maintienne et fasse acte de transmission. 
Et ce fut la Chine, sa rencontre avec Huodatong à Chengdu, où il se rend de très nombreuses fois pour transmettre la psychanalyse à la Freud et à la Lacan. Sans s'illustrer d'un quelconque esprit de conquête dont on est abreuvé en France dans la communauté des psy. Il y part la première fois avec dans sa valise le film Shoah de Lanzmann qui est un des appuis de Psychanalyse Actuelle. Il le montre. C'est la rencontre immédiate sur la destructivité en acte en Chine. Et qui évoque tellement celle de l'Europe que le travail de transmission devient réciproque. Preuve en est : Michel Guibal désigne passeur Huodatong son analysant.

Voilà l'intelligence de la position de Michel où il agit son savoir d'analyste pour ouvrir de nouvelles voies pour transmettre nos enjeux de praticiens de l'inconscient. Il était aussi fin cuisinier de mets de son coin de France qu'il aimait tant, le Gers le pays de ses parents, d'où il tirait sans bruits sa force de vivre.

Michel tu nous quittes, mon ami, mon grand ami...

Jean-Jacques Moscovitz (Psychanalyse actuelle)

\section{We Hommage à Monsieur Michel Guibal}

\footnotetext{
' 'ai commencé à faire une analyse chez Michel Guibal en septembre 1987. Comme mon français est très mauvais, dans certains cas je n'arrive pas à trouver ( le juste mot français pour traduire le caractère chinois auquel que je pense. Il m'a demandé done d'écrire le caractère sur le papier et d'expliquer la structure de ce caractère chinois et d'expliquer la signification des composants de ce caractère. Mais, dans beaucoup de cas, je ne comprends pas la cause de la construction des ces caractères chinois.

Cela m'a fait honte, parce que j’ai une formation en histoire chinoise et j’ai travaillé, en tant qu'historien, pendant cinq ans au Musée du palais impérial à Beijing. Cela m'a conduit à commencer à étudier l'histoire de l'écriture et sa structure.

Les plus anciens caractères chinois sont les pictogrammes. Ces pictogrammes ont une double fonction. Ils représentent le signifié et aussi le signifiant. C'est-à-dire que le pictogramme est aussi le phonogramme.
} 
Par une évolution, les pictogrammes sont devenu les idéophonogrammes. Ils comprennent principalement deux parties dont une partie, qui est idéographique, représente le signifié et l'autre partie, phonographique, représente le son et le signifiant.

La double structure de l'écriture chinoise peut nous aider à résoudre l'opposition qui se trouve entre la théorie de l'inconscient freudien et celle de l'inconscient lacanien. Car Freud insiste toujours que l'inconscient comporte seulement les représentations de chose, c'est-à-dire les signifiés ; tandis que Lacan pense toujours que l'inconscient est structuré comme un langage, c'est-à-dire l'inconscient comporte seulement les signifiants ou bien les représentations de mots.

Et la double structure de l'écriture chinoise nous conduit à penser que l'inconscient a deux dimensions (si on ajoute l'émotion, il a trois dimensions) dont l'une est la représentation de chose, le signifié, c'est la dimension freudienne, et l'autre, la représentation de mot, le signifiant, c'est la dimension lacanienne.

À partir de l'année 2000, il commence à venir régulièrement à Chengdu pour donner un séminaire ou bien pour organiser le colloque. Il fait aussi venir à Chengdu beaucoup de ses amis psychanalystes français. Il a fait tout ce qu'il est possible pour la diffusion de la psychanalyse en Chine, pour le développement de la psychanalyse en Chine et pour l'établissement de l'école chinoise de psychanalyse.

Nous, Centre psychanalytique de Chengdu, comptons ériger une tombe qui contient seulement ses effets personnels à Chengdu et publier un numéro spécial delà revue Étude de la psychanalyse qui contient tous les textes des séminaires qu'il a tenus à Chengdu.

Je souhaite le plus sincèrement qu'il repose en paix.

Huo Datong

\section{We Pour Michel Guibal}

'était une fin de matinée, à Amiens. Je reçus un coup de téléphone, gorgé des sanglots de mon ex thésarde Xu Dan, de la nouvelle de la mort de Michal Guibal. J'étais sur le point de sortir d'un taxi devant parler à un colloque sur le corps du jazz. Je n'ai que peu de souvenir de ce que j’ai pu dire à ce moment là, si ce n'est que les volutes du saxophone de Lester Young, la raucité de Bessie Smith, 
la modalité étirée et disjointe de Coltrane s'entrelaçaient comme une calligraphie chinoise. Je fis à trois reprises ce lapsus insistant entre chorégraphie et calligraphie. Façon sans doute que j'avais, dans un pré deuil, de mettre en résonnance, de faire danser ensemble deux univers autres et pour moi nécessaires, le Jazz et ce que j’ai pu connaître de l'écriture et de la culture chinoise, grâce à Michel Guibal, lui qui m'a ouvert les porte de ce Céleste Empire, me conviant en compagnie de tout un contingent de psychanalystes, droit issus de l'Inter-Associatif européen de Psychanalyse, à Chengdu, en 2004.

Mais commençons par un commencement. Avant que l'amitié que me portait Michel Guibal m'entraîna au large, Guibal était pour moi un nom. Celui du signataire d'un livre organisé avec quelques autres (dont P. Lévy, A. Cherki, R. Zygouris) sur la thématique "Étranger: Crise/représentation ». Je n'avais pas encore connu l'épreuve du divan, la psychanalyse me fascinait, Lacan surtout, mais tout cela me semblait un peu lointain. La façon dont avec culture et simplicité aussi, sans la moindre esbroufe, ces psychanalystes parlaient de l'étranger me toucha au plus vif. C'est sans doute cette lecture qui me guida, sans même que j'en soupçonnasse alors l'effet, lorsque je fis choix et de m'autoriser de mon diplôme tout frais de psychologue clinicien pour trouver un poste en hôpital psychiatrique, à Ville-Évrard lieu où je travaille encore.

Je rencontrai enfin Michel Guibal, là où il parlait, dans le cadre de l'association Psychanalyse actuelle. La psychose, l'autisme (avec R. Abibon), la question centrale du miroir, de la motricité, de l'hallucination, du sonore structurant, tels étaient les thèmes sur quoi sa parole rebondissait. Voix chaude, complice, rocailleuse parfois, crépitant d'intelligence et de malice Références à Lacan mais ausi et surtout à P. Aulagnier et G. Pankov, dont je me mis à travailler autrement les textes.

Les années passant, les liens se tissèrent. Michel Guibal aida Huo Datong, son analysant chinois, promu star parisienne et professeur de philosophie à l'université du Sichuan, à venir parler à Paris devant un parterre de psychanalystes. Il s'est dit tant d'âneries sur les séances entre Michel et Huo Datong, que je mentionne que les séances ne se déroulèrent pas en anglais, et je le redis car Michel fut de longues et longues années encore profondément peiné par les billevesées qui ont pu être écrites et colportées à ce propos. L'un et l'autre de nos deux protagonistes s'aventurèrent dans la langue qui lui était étrangère ou peu familière.

Il ne s'agit pas en ce quelques lignes de retracer toute l'importance qu'eut pour nos amis de Chengdu (et dans une moindre mesure de Pékin ou de Xian) la présence constamment attentive et bienveillante, généreuse ô combien, de Michel Guibal. J'admire qu'il se soit laissé enseigner là-bas. Qu'il ne se soit pas posé en position de maître d'école ou de missionnaire au rabais enseignant la psychanalyse à ces drôles de sujets du lointain.

Psychanalyse réciproque et anthropologie réciproque, non mimétique, certes, mais respectant la disparité du transfert sans la rabattre sur une relation pédagogique et 
paternaliste. Il fallait pour cela une intrépidité, une éthique et une belle dose d'humour. Michel Guibal conjugue pour moi la vertu de l'étonnement à la grâce de l'émerveillement. Il aimait bricoler en chinois avec sérieux, sans pontifier le moins du monde. Voilà comment je perçois un peu de son élan; attentif à ce qu'ont de dynamique, de vivant d'instable et d'ouvert plus qu'on le dit, par peur ou paresse, les constructions des psychoses; heureux d'entendre comment lettre, corps et voix, dansent ensemble autrement dans un monde lointain, Michel est un homme du voyage accompli, de l'errance féconde. Il fonde, non dans l'envie d'occuper la fonction prestigieuse de celui par qui s'ouvrent de grands commencements, mais dans l'effet de cet élan qui l'a porté et le portait encore alors qu'il était souffrant et hospitalisé.

Mon dernier souvenir est le suivant. Autour de son lit d'hôpital Jean-Jacques Moscovitz, Patrick Belamich et moi. Nous devisions en douce amitié. Nous étions presque chez nous. Amertume minimale. Rires.

Mais permettez moi de ne pas conclure ce portrait encore un peu flou sur une note douce-amère, mais par un éclat de joie. Je me souviens encore de Michel, heureux comme un enfant ayant découvert une perle roulant dans le chemin, me parlant avec tant de chaleur riante d'un extrait de l'œuvre d'un taö̈ste tardif, Wang Chong, La balance des discours. Le voici : “ Ni Yue excellait dans l'art de défaire les nœuds, rien ne lui résistait et pourtant il se trouva sans recours face à un noud inextricable. Non qu'il manquât d'ingéniosité, c'est le nœud lui-même qui ne pouvait être défait; et s'il est néanmoins parvenu à la défaire, c'est pour avoir compris que le noud était inextricable. » Et Michel de m'expliquer que c'était le même caractère qui selon lui désignait le nouage et le dénouage.

De l'écriture chinoise à la topologie il n'y avait pas pour Michel passage ou rupture comme ce fut peut-être le cas pour Lacan mais co-présence. Comment l'humain écrit-il l'inconscient et l'image inconsciente du corps? Une telle question est aussi urgente que redoutable, nous n'y échappons pas, mais nous pouvons la féconder dans nos trajets, notre travail d'analyste, notre écoute, notre façon de se laisser entamer par l'autre, sans trop se croire séduit ou dépossédé.

C'est cette position qui permet que des êtres, que tout le culturalisme pose comme étant d'abord différents, peuvent et veulent, à l'encontre de ce qui les assignerait à un destin clos, se déborder d'eux-mêmes et de leurs certitudes. Non pour se faire autre, mais pour laisser vire l'autre en soi et hors de soi.

Merci Michel Guibal.

Olivier Douvile 